\title{
Relación entre la población de adultos de Premnotrypes vorax (Hustache) al inicio del cultivo de papa y el daño de tubérculos a la cosecha
}

\section{Patricio Gallegos G.*}

\begin{abstract}
RESUMEN
El trabajo se realizó para conocer la relación existente entre la población de adultos de Premnotrypes vorax al inicio del cultivo con el grado de daño causado en los tubérculos a la cosecha, y además con el número de aplicaciones de un insecticida específico (Carbofuran G)

El muestreo del número de adultos de $P$. vorax presentes en la etapa comprendida entre los 30 días antes de la siembra hasta los 30 días posteriores y las de 40 y 50 días después de la siembra se correlacionaron en forma significativa $(\mathrm{p}=0.05)$ con el porcentaje de tubérculos dañados a la cosecha, con índices de $0.48,0.44$ y 0.43 respectivamente.
\end{abstract}

La distribución de los adultos entre tratamientos no presentó variación estadística a pesar de que varios de ellos sí recibieron Carbofuran G. Esta información indica que el insecticida no afectó a los adultos, pero sí permitió diferencias de dañe por larvas en tos tubérculos.

Las aplicaciones de Carbofuran $\mathrm{G}$ a la emergencia, al aporque, a la emergencia + aporque, y a la siembra + emergencia + aporque, presentaron 11.8 a $18.2 \%$ de tubérculos con daño, y el testigo 42.6\%. Los tratamientos que recibieron insecticida se encuentran en un mismo rango según Tukey y el testigo en otro rango.

En razón de que los tratamientos que recibieron insecticida no son estadísticamente diferentes es preferible, dado el nivel de la plaga, la aplicación en una sola oportunidad, que las aplicaciones en dos o tres oportunidades.

La población en las diferentes etapas del ensayo varió de 35 a 67 adultos por trampa.

\section{Palabras Claves adicionales: $\quad$ trampas, Carbofuran, correlaciones}

Aceptado para publicación: marzo 22, 1996

* Ing. Agr. M.C. Dto. de Protección Vegetal, E.E. Santa Catalina, INIAP. Casilla 340. Quito, Ecuador. 


\section{Relation between Adult Population of the Premnotrypes Vorax (Hustache) at the Initial Stages Of the Potato Crop Development and Tuber Damage At Harvest}

This study carried out to asses the relationship between the number of Premnotrypes vorax adults at the beginning of the potato crop growth and 1 . the percentage of damage tubers at harvest and 2. the number of insecticide applications.

The adult population among treatments showed no statistical differences i.e. the insecticide had no effect in adults but it affected the larvae activity. The population of $P$. vorax between 30 days before until 30 days after planting as well as 40 and 50 days after planting, showed significant correlation $(\mathrm{P}=0.05)$ with the percent of damaged tubers. The correlation index were $0.48,0.44$ and 0.43 , respectively.

Carbofuran $\mathrm{G}$ applied at, 1. emergency of plants; 2. before hilling; 3. at emergency and before hilling; and 4 . at planting, emergency and before hilling, showed from 11.8 to $18.2 \%$ of damaged tubers, and the control (without insecticide) $42.6 \%$. The treatments with insecticide were located in the same Tukey range and control in another range. As there was no statistical differences between different number of insecticide applications (according to the amount of adults better to apply Carbofuran only once instead of two or three times. The number of adults ranged from 35 to 67 per trap.

Additional index words: traps, Carbofuran, correlations.

Los productores de papa en el Ecuador reconocen la peligrosidad de Premnotrypes vorax mediante la cuantificación del daño en las etapas finales del cultivo o a la cosecha, momento en el cual ya no es factible realizar ninguna labor de control, con excepción del corte de follaje para acelerar la maduración del tubérculo.

El muestreo de adultos de $P$. vorax mediante trampas, previa a la siembra y en los primeros estados de desarrollo del cultivo puede ser un método para predecir el grado de daño que ocasiona la larva a los tubérculos cosechados. Por otra parte, la magnitud de esta población puede determinar el número de aplicaciones de insecticida para su control y la mejor época de acuerdo al 
desarrollo del cultivo. Un buen producto químico para esta plaga es Carbofuran aplicado en forma granulada.

El objetivo del presente trabajo fue determinar la relación entre la población de adultos previamente a la siembra y en las etapas iniciales del cultivo con el grado de daño en los tubérculos a la cosecha, así como también con el número de aplicaciones y la época del control químico de $P$. vorax.

\section{MATERIALES Y MÉTODOS}

El cultivo de papa en que se realizó este estudio se implantó bajo un diseño de bloques completos al azar con cinco repeticiones, en el que los tratamientos fueron las etapas de uso de Carbofuran G $1.25 \mathrm{~kg}$ de ia/ha; las aplicaciones efectuadas al suelo en las siguientes etapas: emergencia, aporque, siembra + emergencia y siembra + emergencia + aporque y un tratamiento sin insecticidas. Además en cada una de estas unidades experimentales se evaluó la población de adultos de P. vorax.

La determinación del número de adultos abarcó el período comprendido entre 30 días antes, de la siembra hasta 50 días después, con observaciones por semana. Los valores obtenidos de la misma en cada oportunidad se sumarizaron por etapas de tiempo, así: las que van desde los 30 y 15 días antes (-) de la siembra y las de hasta los 30, 40 y 50 días después (+) de la siembra.

La evaluación del número de insectos fue posible mediante el uso de trampas, las que consistieron de plantas de papa de aproximadamente $30 \mathrm{~cm}$ de largo, colocadas sobre el suelo y cubiertas por un cartón de 40 x 40 cm; estos insectos no se eliminaron a fin que continuarán con vida en el área de cada parcela. En el punto central de cada unidad experimental se colocó una sola trampa, antes de la preparación del suelo en un sitio aproximado y después en el lugar exacto.

El efecto de los tratamientos se determinó a la cosecha en base al porcentaje de daño de la larva de $P$. vorax.

\section{RESULTADOS Y DISCUSIÓN}

Los primeros trabajos para determinar el número de controles químicos para $P$. vorax se basan en el conocimiento del porcentaje de daño en los tubérculos del ciclo inmediato anterior (4), sin embargo este método no considera los casos en los que hay un cultivo de rotación o barbecho. 
Por otra parte, el estudio de la fluctuación de la población de adultos, (1) y del Control integrado de $P$. vorax mediante el manejo de la población de adultos previa a la siembra (2 y 3) permitieron demostrar la importancia de la presencia del insecto adulto en las etapas iniciales del cultivo.

Los resultados del presente estudio permiten indicar lo siguiente:

El análisis de varianza para los valores de captura de adultos en cada una de etapas de evaluación estudiadas no presentó significación estadística (Tabla 1), lo que demuestra que los adultos se distribuyeron uniformemente en toda el área. Por lo tanto no hubo efecto de la aplicación del insecticida Carbofuran granulado sobre estos adultos.

Tabla 1. Número de adultos de P. vorax capturados por trampa en cinco etapas (días). Santa Catalina, Ecuador, 1994.

\begin{tabular}{lccccc}
\hline Epoca de uso & \multicolumn{5}{c}{ No de adultos } \\
\cline { 2 - 6 } Carbofuran G & \multicolumn{5}{c}{ Etapas en días en base de la fecha de siembra } \\
\cline { 2 - 6 } & -30 & -15 & +30 & +40 & +50 \\
\hline & & & & & \\
Emergencia (E) & 4.8 & 2.0. & 38.8 & 47.2 & 53.2 \\
Aporque (A) & 7.4 & 1.6 & 27.2 & 36.2 & 39.6 \\
E+A & 11.8 & 3.2 & 37.8 & 53.4 & 60.2 \\
Siembra+E+A & 8.8 & 2.2 & 31.4 & 35.2 & 36.2 \\
Testigo & 10.0 & 2.8 & 44.6 & 57.4 & 63.0 \\
Significación & ns** & ns & ns & ns & ns \\
\hline
\end{tabular}

* Carbofuran G 1.25 kg ia/ha

** Prueba de F $(\mathrm{P}=0,05)$

El análisis de varianza de los valores de los tubérculos con daño del gusano blanco, en relación con las etapas de aplicación de Carbofuran G, mostró alta significación estadística, indicándonos que al menos una de ellas fue diferente ( $\mathrm{P}=0.05)$; el coeficiente de variación fue de $25 \%$. La prueba de significación de Tukey, señaló la presencia de dos rangos, en uno de ellos constan todos los tratamientos de aplicación de Carbofuran y en el otro el testigo sin insecticida (Tabla3).

El análisis de correlación entre las etapas de captura de adultos de $P$. vorax y el porcentaje de tubérculos con daño a la cosecha se presenta en la Tabla 2. 
Las poblaciones de adultos que se correlacionaron significativamente con el daño de los tubérculos a la cosecha fueron las de 40 y 50 días después de la siembra y la del período desde los 30 días antes de la siembra hasta los 30 días después de la siembra. La importancia de la significación para las correlaciones a los 40 días después de la siembra radica en que brinda las mejores oportunidades para realizar las medidas de control de la plaga en relación al desarrollo del cultivo y a las prácticas culturales.

El sistema de trampas permite capturar de mejor manera a la población de adultos hasta la emergencia del cultivo, luego de lo cual compiten con las plantas, razón por la cual se extendió el muestreo únicamente hasta los 50 días después de la siembra.

La Tabla 3 permite comparar los tratamiento de fechas de aplicación de Carbofuran y los valores de la población de adultos en las etapas que presentaron correlación estadística significativa entre población de adultos y daño, con el porcentaje de tubérculos con daño causado por larvas.

La Tabla 3 indica que con niveles de población de adultos de 35 a 60 en cualquiera de las tres etapas de evaluación de adultos, la aplicación de Carbofuran en una sola oportunidad, Emergencia o Aporque, presentó valores de daño estadísticamente semejantes a los de dos y tres aplicaciones. Los niveles de población de 57 a 67 adultos en el testigo, en el que no se aplicó insecticida, registró $42.6 \%$ de tubérculos con daño. Cabe señalar que con más de $30 \%$ de tubérculos con daño difícilmente se logra comercializar la papa.

Tabla 2. Etapas de captura de adultos de $P$. vorax que comprenden días antes (-) y después (+) de la siembra, y la correlación entre población de adultos y daño de las larvas en tubérculos. Santa Catalina, Ecuador 1995.

\begin{tabular}{cc}
\hline Etapas de captura de adultos P. vorax & Indice de correlación \\
\hline-30 & $0.223 \mathrm{~ns}$ \\
-15 & $-0.272 \mathrm{~ns}$ \\
+30 & $0.383 \mathrm{~ns}$ \\
+40 & $0.436 *$ \\
+50 & $0.431^{*}$ \\
$-30+30$ & $0.481^{*}$ \\
\hline
\end{tabular}

* Carbofuran $1.25 \mathrm{~kg}$ ia/ha. 


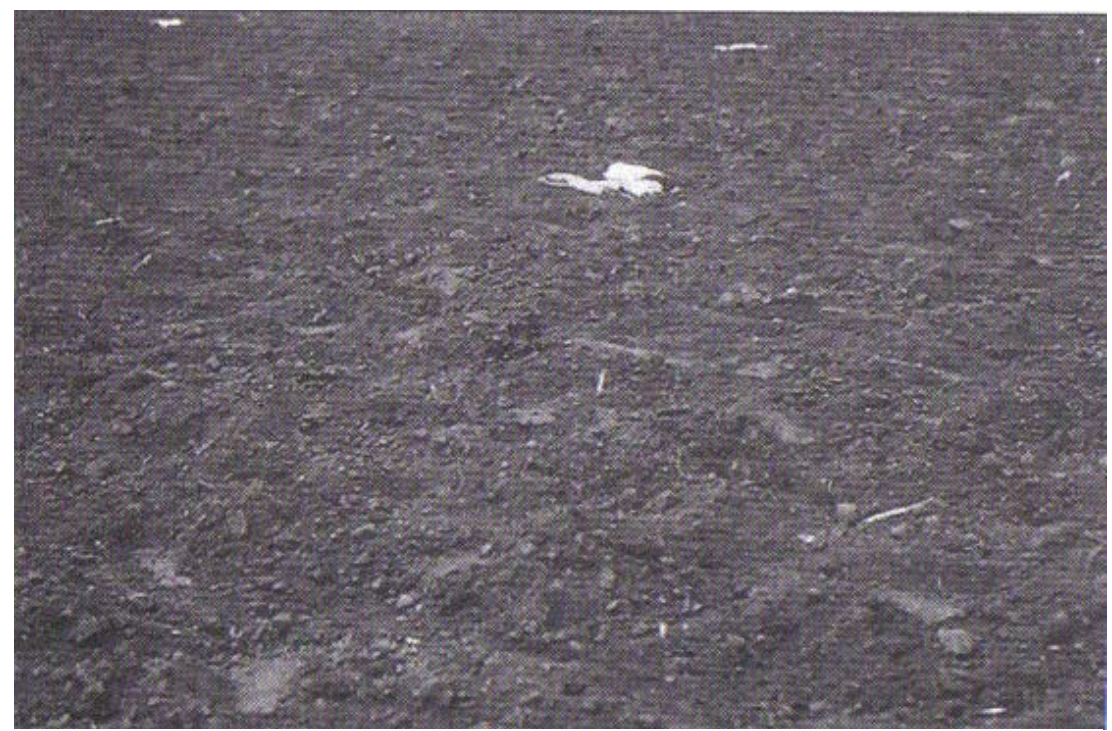

Figura 1.Planta Trampa con 821 adultos de P vorax, capturados en 5 días

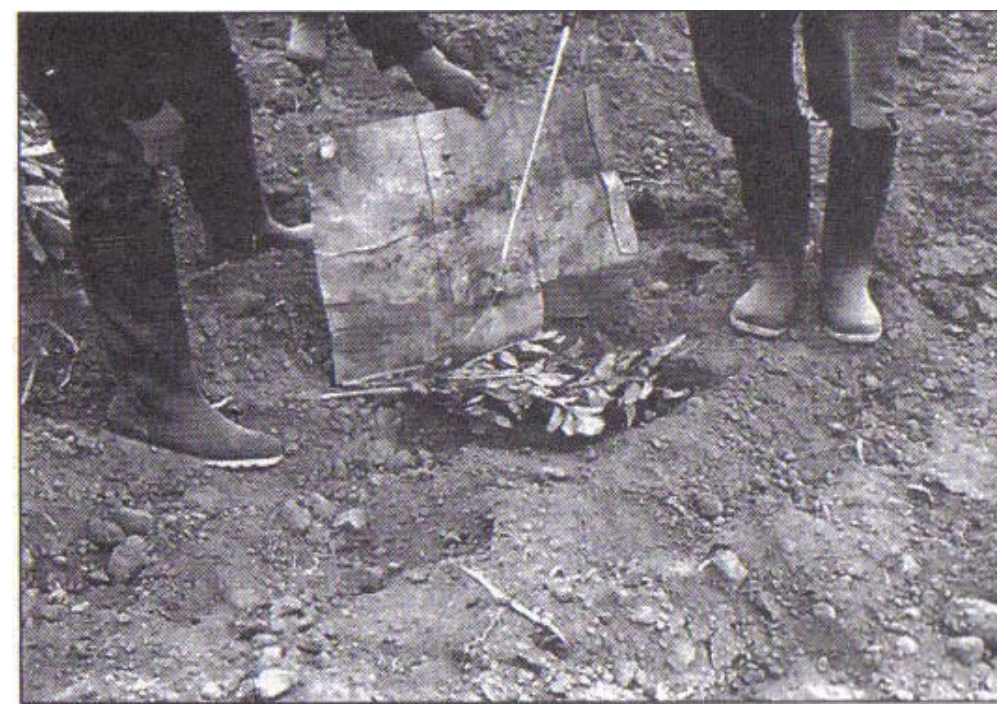

Figura 2. Trampas y plantas cebo en campo de un agricultor para i adultos de $P$. vorax, antes de la siembra del cultivo. 
Tabla 3. Épocas de uso de Carbofuran, número de adultos en las tres etapas de captura que presentaron significación en la correlación con daño y el porcentaje de tubérculos con daño de $P$. vorax. Santa Catalina, Ecuador, 1995.

\begin{tabular}{|c|c|c|c|c|c|}
\hline \multirow{3}{*}{$\begin{array}{l}\text { Época de uso } \\
\text { Carbofuran } \mathrm{G}^{*}\end{array}$} & \multicolumn{5}{|c|}{$\mathrm{N}^{\circ}$ de adultos de $P$. vorax en tres } \\
\hline & \multicolumn{3}{|c|}{ etapas captura (días) } & \multicolumn{2}{|l|}{$\%$ de } \\
\hline & $-30+30$ & +40 & +50 & con dañ & \\
\hline Emergencia (E) & 46 & 47 & 53 & 18.2 & $\mathrm{a}$ \\
\hline Aporque (A) & 36 & 36 & 40 & 18.6 & $\mathrm{a}$ \\
\hline $\mathrm{E}+\mathrm{A}$ & 53 & 53 & 60 & 11.6 & $\mathrm{a}$ \\
\hline Siembra + E + A & 42 & 35 & 36 & 11.8 & $\mathrm{a}$ \\
\hline Testigo & 67 & 57 & 63 & 42.6 & $\mathrm{~b}$ \\
\hline
\end{tabular}

* Carbofuran $1.25 \mathrm{~kg}$ ia/ha.

\section{CONCLUSIONES}

1. Las poblaciones de adultos de $P$. vorax desde 30 días antes de la siembra hasta 30 días después y la de los 40 y 50 días después de la siembra se correlacionaron significativamente con el daño de los tubérculos a la cosecha.

2. Las correlaciones más útiles son aquellas que brindan mayor oportunidad en tiempo para la aplicación de medidas de control, como son desde 30 días antes de la siembra hasta los 40 días de edad del cultivo.

3. Las aplicaciones de Carbofuran granulado en las diferentes etapas del cultivo no afectaron a la población de adultos de $P$. vorax, pero sí al daño de las larvas en los tubérculos a la cosecha.

4. El número de controles químicos puede ser inferior al máximo de tres, dependiendo de la población de adultos presentes en el área.

5. Es factible el pronóstico del daño de los tubérculos a la cosecha con base en la población de adultos en el período comprendido entre los 30 días antes y 50 días después de la siembra.

6. Es necesario generar un modelo que permita predecir el daño a la cosecha bajo diferentes niveles de población de la plaga. 


\section{REFERENCIAS BIBLIOGRÁFICAS}

1. Gallegos, P. 1993. Fluctuación de la población de adultos de Premnotrypes vorax en el cultivo de la papa. Resúmenes del Congreso latinoamericano de la Papa. Santo Domingo, República Dominicana.

2. Gallegos, P. 1993. Control integrado Premnotrypes vorax mediante manejo de la población de adultos y control químico en el cultivo de la papa. En: Informe Anual Proyecto INIAP-FORTIPAPA 1992-1993. Subproyecto 3.1.6, Quito, Ecuador.

3. Gallegos, P. 1994. Control integrado de Premnotrypes vorax mediante manejo de la población de adultos y control químico en el cultivo de la papa. En Informe Anual Proyecto INIAP-FORTIPAPA 1993-1994. Subproyecto 3.1.6, Quito, Ecuador.

4. Merino, G.; Vásquez, V. 1979. Recomendaciones preliminares para determinar el grado de daño del gusano blanco de la papa y su control. INIAP Boletín Divulgativo No. 108. Quito, Ecuador 